\title{
Programa Saúde da Família e as ações em nutrição em um distrito de saúde do município de São Paulo
}

\author{
The Family Health Program and nutritional activities \\ in a health district in São Paulo, Brazil
}

Priscila M aria Fúncia Fernandez 1

Silvia M aria Voci 1

Lúcia Hitomi Kamata 1

M yrian Spínola Najas 1

Ana Lúcia M edeiros de Souza 1

\footnotetext{
1 Departamento de M edicina Preventiva da UNIFESP/EPM. Rua Pedro de Toledo 675, 04039-032, São Paulo SP. pmfuncia@usp.br
}

Abstract To overcome the guidelines of Family $\mathrm{H}$ ealth Program in São Paulo city, the H ealth's local O rganization had defined priority areas for the teams' implementation, based on the social exclusion map, the social-economical and epidemiological profile of the health district. In the Vila M aria Health District, teams from this program were implanted in the Parque N ovo M undo region. Because there is no training in the field of food and nutrition actions for these professionals, the work was developed, conducting visits at territory, despite surveys from the epidemiological and social-economical profileand interviews. The training was divided in four classes having different approaches, related with nutritional diagnostic and mains transmissible chronical and scarcity diseases controlled by diet (by the nutrition). Therefore, it was possible to denay the beliefs on wrong behaviors, as well as to show the possibility to the application of this information to the community, for all the teams' professionals.

Key words Family Health Program, Training, Nutrition, Education public health professional
Resumo Com o objetivo de operacionalizar as diretrizes do Programa Saúde da Família no município de São Paulo, a Secretaria M unicipal de Saúde definiu áreas prioritárias para a implantação das equipes, baseando-se no $M$ apa de Exclusão Social e no perfil socioeconômico e epidemiológico dos Distritos de Saúde. As equipes do Programa foram implantadas em áreas onde havia bolsões de pobreza. Por não haver treinamentos nas ações de nutrição e al imentação para esses profissionais, foi elaborado o presente trabalho. Realizaram-se entrevistas com os integrantes das equipes, visitas ao território e levantamento do perfil epidemiológico e socioeconômico. 0 treinamento foi dividido em quatro aulas, elaboradas com enfoque diferenciado de acordo com a atribuição de cada profissional. A partir do treinamento, foi possível reverter condutas equivocadas, bem como mostrar a possibilidade de aplicação dessas informações na comunidade, por todos os profissionais integrantes das equipes.

Palavras-chave Programa Saúde da Família, Capacitação, Nutrição, Educação profissional em saúde pública 


\section{Introdução}

A implantação do Programa Saúde da Família (PSF) tem como objetivo geral melhorar o estado de saúde da população, mediante a construção de um modelo assistencial de atenção, baseada na promoção e proteção, no diagnóstico precoce, na recuperação da saúde dos indivíduos e da família, de forma integral e contínua de conformidade com os princípios e diretrizes do Sistema Ú nico de Saúde (SU S): universalização, descentralização, integralidade e participação da comunidade (M S, 2001).

Em São Paulo, as equipes do PSF foram implantadas em áreas de maior prioridade, de acordo com o M apa de Exclusão Social, através de critérios socioeconômicos e epidemiológicos (Prefeitura de São Paulo, 2002).

$N$ a rede de apoio, envolvida na implantação do PSF, podem ser citadas parcerias com instituições de ensino superior. A Universidade Federal de São Paulo/ Escola Paulista de M edicina (UNIFESP/EPM) participa como parceira, sendo conveniada com os Distritos de Saúde de Vila M aria (DSVM) e o de Sacomã. Também chamados de "Distrito Escola", neles alunos de graduação e pós-graduação desenvolvem projetos para a melhoria das condições de saúde destes locais.

As equipes do PSF são responsáveis pela população residente em um território definido. As famílias são cadastradas e, com base nestas informações, cada equipe realiza o diagnóstico local sobre as condições sociodemográficas. A partir da identificação dos problemas de saúde prevalentes e situações de risco, é elaborado um planejamento de atuação das equipes.

Observada a necessidade de aprimorar e padronizar os conceitos e condutas nutricionais, de acordo com a demanda desta população, elaborou-se um treinamento, com o objetivo de capacitar os profissionais do PSF, nas ações de alimentação e nutrição.

\section{Metodologia}

\section{Área de estudo}

O DSVM écomposto pelos Distritos Administrativos de Vila M aria, Vila Guilherme eVila M edeiros, localizando-se na região norte do município de São Paulo. Caracterizam-se por condições de vida heterogêneas, destacando-se o Parque N ovo M undo (localizado no Distrito
Administrativo de Vila M aria), que apresenta uma população empobrecida, condições de saneamento precárias, grandes favelas e regiões chacareiras, sendo esta região a selecionada para a implantação das equipes de PSF neste distrito (SM S, 2002).

$\mathrm{Na}$ elaboração do treinamento, realizaramse visitas de abril a maio de 2002 para se reconhecer a localidade de estudo; foram efetuadas entrevistas com as equipes para o levantamento das condições de saúde da população e do perfil epidemiológico, utilizando-se, também, fichas cadastrais do cartão SUS e dados oficiais do Instituto Brasileiro de Geografia e Estatística (IBGE) e da Secretaria M unicipal de Saúde do município de São Paulo (SM S, 2002; IBGE, 2003).

De acordo com este levantamento, foram cadastradas no Parque Novo Mundo I e II 7.561 famílias, totalizando 28.634 pessoas atendidas. Nos dois locais, observaram-se distribuições semelhantes em relação ao sexo e à faixa etária; $52 \%$ da população era do sexo feminino, com maior concentração na faixa etária de 20 a 39 anos (aproximadamente $37 \%$ ) e em menores de 10 anos (aproximadamente 26\%).

Em relação à escolaridade, 97,7\% e 95,6\% dos indivíduos da faixa etária de 7 a 14 anos estavam na escola e $84,3 \%$ e $87,7 \%$ daqueles com 15 anos ou mais eram alfabetizados nos Parques N ovo M undo I ell respectivamente.

Q uanto ao saneamento básico, as famílias contavam com abastecimento de água por rede pública, mas grande parte delas não tratava a água no domicílio (processos de filtração, fervura ou cloração).

No Parque N ovo M undo I, 97,8\% das famílias tinham o serviço de coleta pública de lixo e $83,3 \%$ das moradias possuíam rede de esgoto, sendo que $16,3 \%$ tinham como destino céu aberto e $0,4 \%$ fossas.

No Parque Novo M undo II, observou-se uma condição mais precária no que se refere ao destino do lixo; $89,8 \%$ das famílias possuíam coleta pública, 10,1\% jogavam o lixo a céu aberto e $0,1 \%$ queimava ou enterrava. Quanto ao destino do esgoto, $68,6 \%$ estavam ligados à rede pública de coleta, $4,4 \%$ fossa e $27 \%$ a céu aberto.

Do total de domicílios do Distrito Administrativo de Vila M aria, 19,1\% localizavam-se nas 11 favelas existentes e 2,5\% eram apartamentos que compunham os dois "Cingapuras" (conjunto habitacional) da região. Observando-se a situação dos domicílios, 8,4\% eram considerados precários, sendo que 7,2\% eram 
barracos de favela, 0,8\% eram cortiços e 0,4\% improvisados.

Levando-se em consideração o tipo de moradia, a maioria era de tijolo ou adobe e a principal doença referida pela população foi a hipertensão arterial, seguida de diabetes mellitus na faixa etária de 15 anos ou mais.

Os profissionais das equipes, quando entrevistados, também relataram a hipertensão arterial e o diabetes mellitus como sendo os problemas de saúde mais prevalentes na população atendida. Foram citados, também, diarréia, parasitoses, anemia, infecções e outros agravantes dos problemas de saúde como: alcoolismo, uso de drogas, gestação na adolescência, alta paridade, ausência do aleitamento materno e desmame precoce. A presença de desnutrição foi informada por poucos profissionais, porém, nas visitas ao território, observou-se a existência tanto desse distúrbio nutricional como de obesidade. 0 conhecimento deste perfil contribuiu para a elaboração do treinamento das equipes do PSF deste distrito, com estratégias mais adequadas à realidade.

Quanto às ações de nutrição, realizadas pelas equipes, foi verificado que as orientações nutricionais mais oferecidas à comunidade estavam relacionadas à hipertensão arterial, diabetes mellitus, higienização de alimentos e alimentação infantil.

Observou-se, também, que al guns profissionais não realizavam as medidas antropométricas por falta de equipamentos nas U nidades ou devido ao elevado número de consultas realizadas pelos médicos, sendo que a maior atenção era dada às gestantes.

Os profissionais manifestaram a necessidade de atualização e padronização das condutas nutricionais, relatando a ausência de um treinamento voltado para a capacitação das equipes em nutrição.

Verificada esta necessidade, elaborou-se um treinamento que foi dividido em quatro encontros, sendo os profissionais agrupados de acordo com o seu grau de instrução. A divulgação foi feita pela coordenação do distrito, e a convocação dos profissionais, publicada no Diário Oficial do M unicípio de São Paulo (2002).

As aulas foram elaboradas, respeitando-se as atribuições específicas de cada profissional. Estes foram divididos em três turmas: a 1a turma, constituída por oito médicos e oito enfermeiras dos Parques N ovo M undo I ell; 2a turma, com 22 agentes comunitários de saúde (ACS) e oito auxiliares de enfermagem do Parque Novo
M undo I; 3a turma, com 23 ACS e oito auxiliares de enfermagem do Parque Novo M undo II. 0 plano das aulas é demonstrado na tabela 1.

\section{Treinamento}

Na primeira aula de treinamento, foram levantados os conhecimentos de nutrição por parte de cada um deles.

No início do segundo encontro, as equipes foram questionadas em relação aos principais conhecimentos que tinham e que comumente a população relatava sobre obesidade, diabetes, hipertensão arterial e dislipidemia. Dessa forma, foi possível maior aproximação da realidade e melhor conhecimento da rotina de cada profissional e dos saberes em nutrição. Essa atividade foi dinâmica, realizando-se as anotações em um painel. Ao final da aula, os conceitos transmitidos foram comparados com os exemplos citados no início, mostrando qual informação era válida e o que poderia ser considerado um conceito ultrapassado.

$\mathrm{N}$ a terceira aula, foi utilizado o mesmo método da aula anterior, porém abordando os temas relacionados com desnutrição, anemia e diarréia. N esta aula, foi realizada uma atividade prática com agentes comunitários e auxiliares de enfermagem, a fim de se padronizar 0 preparo do soro caseiro.

$\mathrm{Na}$ última aula, os profissionais (médicos, enfermeiras, auxiliares de enfermagem e ACS) dividiram-se de acordo com as suas equipes de atuação no PSF e UBS, para que trabal hassem os conceitos discutidos nos três encontros anteriores, de forma integrada.

Foi proposta a análise de um estudo de caso, abordando uma família composta de dois adultos, uma criança e um idoso. Cada equipe recebeu o relato do caso com um enfoque maior em um dos membros da família. Ao final, foram apresentadas as condutas e as observações, utilizando-se tarjetas de cartolina fixadas na parede.

As principais características, descritas no estudo de caso, foram as condições de moradia (casa de alvenaria, sem serviço de água e esgoto, próximo a um córrego) e composição da família (idosa de 67 anos, aposentada, anêmica, desnutrida; mulher de 29 anos, faxineira, hipercolesterolêmica, eutrófica; homem de 33 anos vigia noturno, diabético, hipertenso, hipertrigliceridêmico e obeso; criança de cinco anos, apresentando episódios de diarréia, desnutrida). Ao final dos casos também era ques- 
Tabela 1

Planejamento das aulas ministradas no Parque Novo M undo I e II - São Paulo 2002.

\begin{tabular}{|c|c|c|c|c|}
\hline Data & $\begin{array}{l}\text { Aula } 1 \\
5 \text { e } 6 / 9\end{array}$ & $\begin{array}{l}\text { Aula } 2 \\
16 \text { e } 19 / 9\end{array}$ & $\begin{array}{l}\text { Aula } 3 \\
23 \text { e } 26 / 9\end{array}$ & $\begin{array}{l}\text { Aula } 4 \\
30 / 9 \text { e } 3 / 10\end{array}$ \\
\hline Tema & $\begin{array}{l}\text { - Importância do } \\
\text { diagnóstico nutricional }\end{array}$ & $\begin{array}{l}\text { - O besi dade e doenças } \\
\text { associadas (H ipertensão } \\
\text { Arterial, Diabetes } \\
\text { M ellitus e Dislipidemias) }\end{array}$ & $\begin{array}{l}\text { - Desnutrição e doenças } \\
\text { relacionadas (Diarréia e } \\
\text { Anemia Ferropriva) }\end{array}$ & $\begin{array}{l}\text { - Avaliação do } \\
\text { treinamento a partir } \\
\text { de discussões de casos }\end{array}$ \\
\hline Objetivo & $\begin{array}{l}\text { - Demonstrar a } \\
\text { importância do } \\
\text { diagnóstico nutricional } \\
\text { e padronizar técnicas } \\
\text { de obtenção de medidas } \\
\text { e critérios de diagnóstico } \\
\text { para cada grupo etário }\end{array}$ & $\begin{array}{l}\text { - Padronizar condutase } \\
\text { orientações nutricionais }\end{array}$ & $\begin{array}{l}\text { - Padronizar condutas e } \\
\text { orientações nutricionais }\end{array}$ & - Avaliar o treinamento \\
\hline Conteúdo & $\begin{array}{l}\text { - Transição nutricional } \\
\text { - Importância do } \\
\text { diagnóstico nutricional } \\
\text { - Estado nutricional } \\
\text { associado a doenças } \\
\text { crônicas e infecções } \\
\text { qualidade de vida } \\
\text { - Técnicas de aferição das } \\
\text { medidas antropométricas } \\
\text { em diferentes grupos } \\
\text { etários e estado } \\
\text { fisiológico } \\
\text { - Padrões de referência e } \\
\text { critérios de classificação } \\
\text { do estado nutricional } \\
\text { - Exercício prático }\end{array}$ & $\begin{array}{l}\text { - Discussão do estado } \\
\text { nutricional e casos } \\
\text { levantados nas áreas de } \\
\text { atuação de cada equipe } \\
\text { - Definição e tipos de } \\
\text { obesidade; Diabetes } \\
\text { M ellitus, H ipertensão } \\
\text { Arterial e Dislipidemia } \\
\text { - Critérios para } \\
\text { diagnósticos destas } \\
\text { doenças } \\
\text { - Condutas nutricionais } \\
\text { - Adaptação das condutas } \\
\text { na população atendida }\end{array}$ & $\begin{array}{l}\text { - Definição de } \\
\text { desnutrição, anemia } \\
\text { ferropriva e diarréia } \\
\text { - Fatores relacionados com } \\
\text { estas doenças } \\
\text { - Valores de referência } \\
\text { para anemia } \\
\text { - Objetivos da intervenção } \\
\text { nutricional } \\
\text { - Tratamento e prevenção } \\
\text { destas doenças }\end{array}$ & - Discussão de casos \\
\hline Estratégia & $\begin{array}{l}\text { - Preleção dialogada } \\
\text { - Demonstração de } \\
\text { técnicas antropométricas }\end{array}$ & $\begin{array}{l}\text { - Preleção dialogada } \\
\text { - Dinâmica para } \\
\text { levantamento de } \\
\text { condutas utilizadas pelos } \\
\text { profissionais }\end{array}$ & $\begin{array}{l}\text { - Preleção dialogada } \\
\text { - Dinâmica para levantar } \\
\text { condutas utilizadas } \\
\text { - Demonstração do } \\
\text { preparo do soro caseiro }\end{array}$ & $\begin{array}{l}\text { - Dinâmica de grupo } \\
\text { envolvendo todos os } \\
\text { profissionais das equipes }\end{array}$ \\
\hline $\begin{array}{l}\text { Recursos } \\
\text { materiais }\end{array}$ & $\begin{array}{l}\text { - Datashow, papel Kraft, } \\
\text { balança digital, } \\
\text { antropômetro de parede, } \\
\text { apostila }\end{array}$ & $\begin{array}{l}\text { - Datashow, papel Kraft, } \\
\text { apostila }\end{array}$ & $\begin{array}{l}\text { Datashow, papel Kraft, } \\
\text { utensílios domésticos } \\
\text { (copo, colheres) e apostila }\end{array}$ & $\begin{array}{l}\text { Datashow, Tarjetas de } \\
\text { cartolina, apostila }\end{array}$ \\
\hline Duração & - 1 hora 30' & - 1 hora 30' & - 1 hora 30' & - 1 hora 30' \\
\hline
\end{tabular}

Obs: Todas as aulas foram ministradas na igreja Santa Rita de Cássia - Parque N ovo M undo. 
tionado qual o papel de cada integrante dentro da equipe.

Essa foi uma forma de realizar a aval iação somativa das equipes, uma vez que, para participar da discussão dos casos, os profissionais teriam de utilizar os tópicos discutidos nas aulas anteriores e acabariam por expor até que ponto os temas abordados foram assimilados.

\section{Resultados}

\section{1a aula}

N otou-se que a avaliação antropométrica era feita por ACS e auxiliares de enfermagem, principalmente em crianças e gestantes, devido à implantação do programa Bolsa Alimentação. Todos os profissionais relataram a necessidade de equipamentos adequados, como 0 antropômetro infantil, pois a maioria das aferições que necessitavam deste instrumento era feita de maneira improvisada.

Deve-se ressaltar que não é, necessariamente, uma obrigação dos ACS realizarem as medidas antropométricas, mas sim, indicar e encaminhar casos para uma avaliação nas UBS.

\section{2ạ aula}

Com a atividade realizada no início da aula, notou-se que muitos já conheciam as condutas a serem seguidas, mas que, muitas vezes, mantinham conceitos ultrapassados, tais como: os diabéticos não podem consumir determinadas frutas e massas; nas dislipidemias, 0 abacate deve ser excluído; na hipertensão arterial, chá de quebra-pedra e chuchu ajudam no seu controle; e na obesidade, alimentos como uva também devem ser retirados.

\section{3a aula}

Em todos os grupos, observou-se o quanto estão presentes os conceitos populares em relação ao tratamento da anemia ferropriva como, por exemplo: "cozinhar em panela de ferro e colocar um prego na hora de cozinhar os alimentos". Portanto, mais uma vez, foi vista a importância de esclarecer e atualizar, principalmente, os médicos e enfermeiros por terem uma grande influência e credibilidade com os usuários do sistema.

\section{4ạ aula}

$\mathrm{N}$ a última aula, todas as equipes do Parque Novo M undo I fizeram o diagnóstico nutricional de forma correta e deram as orientações nutricionais pertinentes aos casos anal isados. Duas, das quatro equipes, apresentaram, além das condutas específicas do caso, orientações para os outros integrantes da família descrita, observando as condições gerais de higiene e analisando a família como um todo, atingindo assim, o objetivo do encontro. Quanto ao papel de cada profissional na equipe, apenas uma conseguiu descrevê-lo, de forma generalizada, não distinguindo funções específicas.

No Parque N ovo M undo II, todas as equipes realizaram o diagnóstico nutricional corretamente. Duas equipes apresentaram orientações dietéticas mais completas, todavia não observaram os demais integrantes da família. Destas, apenas uma respondeu à questão referente ao papel de cada integrante da equipe. As demais observaram toda a situação familiar, sendo que uma em especial salientou práticas saudáveis relacionadas ao estilo de vida e à necessidade de estrutura de apoio social contribuindo para melhora do quadro de saúde. Também foi capaz de descrever todos os profissionais como responsáveis pelo reforço das orientações dadas e acompanhamento das famílias.

A maioria das equipes conseguiu visualizar a família como um todo, de tal maneira que, ao cuidar de um caso em especial, foi capaz de observar e intervir em problemas de saúde de outros integrantes da família, além de observar 0 contexto em que a família estava inserida, acrescentando orientações a outros fatores que influenciavam o estado nutricional, tais como: saneamento, higiene, educação, atividade física e estresse.

Quanto à participação dos profissionais nas aulas, foi notada maior ausência entre os médicos e enfermeiras nos dois últimos encontros, o que pode ter sido uma limitação do treinamento, já que o objetivo do mesmo era padronizar as condutas nutricionais em toda a equipe do PSF e não apenas em alguns profissionais. Provavelmente, estas faltas possam ser atribuídas ao agendamento de outras atividades, no mesmo período do treinamento.

Através de uma avaliação final, verificou-se que a aceitação do treinamento foi positiva, sendo este considerado dinâmico, rápido, explicativo, de fácil compreensão e interativo (principalmente o último encontro), abrangendo aspectos biopsicossociais que se enquadram à realidade, o que é importante no auxílio à comunidade. Foram sugeridas mais aulas ou palestras, com maior freqüência, abordando 
novos assuntos e, se possível, abrangendo toda a comunidade.

\section{Considerações finais}

A implantação do PSF vem acontecendo de maneira diferente em todo o Brasil, sendo um modelo que funciona muito bem em cidades pequenas, as quais, antes de sua implantação, contavam com uma estrutura precária e pouco acesso à saúde, quando comparadas com grandes metrópoles.

Em estudo realizado no município de Florianópolis (SC), técnicos e gestores entrevistados apontaram al guns pontos problemáticos na implantação do PSF como, por exemplo, a dificuldade de obter médicos com formação adequada, visto o número reduzido de residências médicas nessa área. Foram citados, também, problemas que se referem à composição etamanho da equipe, apontando-se a necessidade da inclusão de outros profissionais (Conill, 2002).

Em relatório preliminar, o M S (1999) demonstra os níveis e estágios diferenciados em que se encontra o desenvolvimento da estratégia Saúde da Família no país. Investigaram-se com os coordenadores estaduais, quais seriam as principais limitações na operacional ização do PSF, sendo apontados: a formação inadequada dos profissionais, o número insuficiente de médicos, a falta de recursos financeiros aliada à dificuldade financeira dos municípios eà falta de entendimento dos gestores.

$\mathrm{Na}$ avaliação do Programa M édico de Família (PM F/PSF), em Niterói (RJ), foi relatada a dificuldade de organização e gestão do sistema local de saúde, por conta da grande procura dos serviços pela população de municípios vizinhos. Também houve problemas de incompatibilidade entre o PM F/PSF e a rede de serviços de saúde convencional, em que 0 aspecto $\mathrm{da}$ falta de isonomia salarial foi um ponto importante de tensão entre os profissionais (Senna \& Cohen, 2002).

$N$ ão são muitos os estudos que analisam o Programa, quanto à sua implantação e adequação em cidades com grande número de habitantes. 0 município de São Paulo tem uma ampla rede de saúde, mas hoje se encontra desestruturada pela forma como foi gerida nos últimos governos. Quando se observa a implantação do PSF, em uma cidade como São Paulo, supõe-se que os resultados não sejam os mesmos observados em outros municípios, devido à or- ganização social eà estrutura dos serviços apresentarem muitas peculiaridades. No distrito onde o treinamento foi realizado, a implantação do PSF vem ocorrendo ainda de forma gradual.

Observaram-se durante o treinamento inúmeras dificuldades relacionadas com capacitação, isonomia salarial, formação médica de caráter especialista (não generalista), profissionais nem sempre tão bem treinados ou sem conhecimento suficiente e vontade social, para atender a população mais carente. Estes desarranjos acabam por gerar problemas organizacionais e impedem o bom andamento do Programa, não conseguindo manter a estrutura proposta.

Em relação ao treinamento, observaram-se diferenças importantes entre ACS e auxiliares de enfermagem das equipes das duas U nidades Básicas de Saúde, tanto na participação durante o treinamento, quanto ao próprio relacionamento entre eles. Em geral, os ACS e auxiliares de enfermagem tiveram uma participação mais efetiva quando comparada à dos médicos e das enfermeiras. $\mathrm{Na}$ tentativa de compreender estas diferenças, é importante ressaltar que ainda na sociedade atual vê-se o papel do médico como central e hegemônico, o que não possibilita maior autonomia e criatividade dos agentes e maior integração da equipe (Almeida \& M ishima, 2001). Segundo Pedrosa \& Teles (2001), em trabalho realizado com equipes do PSF em Teresina $(\mathrm{PI})$, quando entrevistados, quanto às relações na equipe, os médicos e a equipe de enfermagem al egavam problemas com os agentes e estes al egavam problemas com médicos e enfermeiras. Situação semelhante pôde ser meIhor observada nas equipes do Parque Novo M undo I, que, no momento, enfrentavam conflitos internos os quais repercutiram, inclusive, no treinamento.

Analisando-se as estratégias dos quatro encontros, observou-se que a dinâmica de grupo, utilizada na avaliação, foi fundamental para relacionar todo o conteúdo do treinamento com uma visão mais crítica do papel de cada membro da equipe, ficando evidente:

- a importância de se avaliar a família como um todo, esclarecendo o valor da atuação de cada um dos profissionais;

- a necessidade de analisar outros aspectos que estão indiretamente relacionados com a saúde;

- que os ACS conhecem melhor os aspectos que envolvem a comunidade e têm muitas informações a respeito de cada família, contri- 
buição fundamental para que médicos e enfermeiras conheçam um pouco mais da realidade de cada paciente;

- a possibilidade dos ACS, auxiliares de enfermagem e enfermeiras participarem das condutas e tratamentos definidos pelo médico;

- queACS, bem treinados, podem solucionar dúvidas simples, diminuindo a demanda de atendimentos da UBS, estimulando e acompanhando de perto o que real mente está sendo seguido e as dificuldades encontradas pelo paciente.

A ênfase maior desse treinamento foi dada aos ACS, devido a todos os fatores acima descritos, para que eles reconhecessem a importância social de seu trabalho, a necessidade de envolvimento e a autonomia.

Essa análise não permitiu avaliar o impacto do treinamento, contudo a reavaliação e a verificação da necessidade de um reforço das infor-

\section{Colaboradores}

PM F Fernandez e SM Voci trabalharam na concepção, planejamento e interpretação dos dados, na elaboração do rascunho, na revisão crítica do conteúdo e na aprovação da versão final do manuscrito; LH Kamata trabalhou na concepção, planejamento e interpretação dos dados, na elaboração do rascunho e na aprovação final do manuscrito; M S N ajas e ALM Souza trabalharam na revisão crítica do conteúdo, na orientação e na aprovação final do manuscrito.

\section{Referências bibliográficas}

Almeida M CP \& M ishima SM 2001. 0 desafio do trabaIho em equipe na atenção à Saúde da Família: construindo "novas autonomias" no trabalho. InterfaceComunicação, Saúde, Educação 5(9):150-153.

Conill EM 2002. Políticas de atenção primária e reformas sanitárias: discutindo a avaliação a partir da análise do Programa Saúde da Família em Florianópolis, Santa Catarina, Brasil, 1994-2000. Cadernos de Saúde Pública 18 (suplemento):191-202.

Diário Oficial do M unicípio de São Paulo 2002. Comunicado 001/02 de 6 de setembro de 2002: comunica a realização do Treinamento sobre enfoque nutricional. Diário Oficial do M unicípio de São Paulo, São Paulo, 6 set.

IBGE (Instituto Brasileiro de Geografia e Estatística) 2000. Censo demográfico 2000: resultado do universo São Paulo. 13 janeiro 2003 <http://www.ibge.com.br>

M S (M inistério da Saúde) 1999. Avaliação da implantação efuncionamento do Programa Saúde da Família - PSF. Relatório preliminar. MS, Brasília. mações transmitidas, nos encontros, podem ser propostas em um futuro trabalho. É preciso reconhecer que, tecnicamente, cada um tem 0 seu conhecimento e suas atribuições. Todavia, "enxergar" um contexto social e compreender as peculiaridades de cada usuário do sistema é extremamente importante e possível de ser feito por qualquer profissional envolvido no PSF. 0 reconhecimento do trabal ho e a concessão de espaço a cada um, dentro da equipe, são necessários para haver maior integração, troca de conhecimentos e a mesma concepção. N em sempre, um mesmo conhecimento técnico deverá ser passado da mesma forma a todos que dele necessitam. É necessário saber adaptá-lo à realidade de cada indivíduo, à sua rotina e à sua capacidade de compreendê-lo de maneira que possa ser aplicado em algum aspecto de sua vida e de sua família.

\section{Agradecimentos}

Às nutricionistas M irian M atsumoto, Giovana, Talita, Ana Paula, Clarice e M ary, pela valiosa contribuição nas diversas etapas deste trabalho; e aos funcionários do Distrito deSaúde de Vila M aria.
MS (M inistério da Saúde) 2001. 0 Programa Saúde da Família - PSF. MS, Brasília.

Pedrosa JIS \& Teles JBM 2001. Consenso e diferenças em equipes do Programa Saúde da Família. Revista de Saúde Pública 35(2):303-311.

Prefeitura do M unicípio de São Paulo, 2002. Programa Saúde da Família. 10 janeiro 2003. Disponível em <http://www5.prefeitura.sp.gov.br/sistema/ bin/pg_dinamica.php?id_pag $=10>$

Senna M CM \& Cohen M M 2002. M odelo assistencial e estratégia saúde da família no nível local: análise de uma experiência. Ciência \& Saúde Coletiva 7(3):523535.

SM S (Secretaria M unicipal de Saúde) 2002. Dados do Consolidado das Famílias Cadastradas por U nidade no ano de 2002. São Paulo: SM S [dados não publicados].

Artigo apresentado em 18/08/2004

Aprovado em 18/01/2005

Versão final apresentada em 18/01/2005 